\title{
Power Constrained Space-Time Processing for Suppression of Electromagnetic Fields
}

\author{
Tommy Hult and Abbas Mohammed
}

\begin{abstract}
Active suppression of noise and vibrations is a well established field of research with many applications in acoustic and mechanical industries. In this paper we investigate the possibility of applying these adaptive active control methods with the aim of lowering the electromagnetic power density at a specific volume in space using the superposition principle and space-time processing employing the full MIMO (Multiple Input Multiple Output) antenna system setup. The application that we evaluated is a model of a mobile phone equipped with one ordinary transmitting antenna and two actuator-antennas which purpose is to cancel out the electromagnetic field at the human head while maintaining a predefined level of the overall output power of the system. This power control is achieved through the use of a quadratic constraint on the active control algorithm. Simulation results show the promise of using the adaptive control algorithms and MIMO system to attenuate the electromagnetic field power density.
\end{abstract}

Index terms: electromagnetic fields, adaptive active control algorithms, multiple-input multiple-output (MIMO) antenna systems, radio wave propagation, power constraints.

\section{INTRODUCTION}

There have been several studies, with conflicting results, on the effects of cell-phone radiation on the human body [1-7]. The amount of radiation emitted from most cell phones is very minute. However, given the close proximity of the phone to the head, it is entirely possible for the radiation to cause harm. The easiest way to minimize the radiation you are exposed to is to position the antenna as far from your head as possible. Utilizing a hands-free kit, a car-kit antenna or a cell phone whose antenna is placed even a couple of inches farther away from the head can do this most effectively. This paper makes a contribution to that discussion by proposing a new approach by exploiting adaptive active control algorithms combined with a MIMO (Multiple Input Multiple Output) antenna system to attenuate the electromagnetic field at a specific volume in

Manuscript received May 16, 2004; revised May 162005 and August 05, 2005. The paper was presented in part at the Conference on Software, Telecommunication and Computer Networks (SoftCOM) 2004.

T. Hult and A. Mohammed are with Department of Signal Processing, Blekinge Institute of Technology, 37225 Ronneby, Sweden (e-mail: \{thu, amo\}@bth.se). space. Active control algorithms for suppression of noise and vibration is a well established field of research with many applications in acoustic and mechanical industries [8-10]. The method of canceling out a signal comes from the principle of superposition. If two signals are superimposed, they will add either constructively or destructively. The main objective of this paper is to investigate the possibility of applying these adaptive active control methods with the aim of lowering the electromagnetic field power density at a specific volume in space using the superposition principle and the full space-time processing (i.e. multiple antennas at both the transmitter and receiver side or MIMO) antenna system setup.

The application we evaluated in this paper is a model of a mobile telephone equipped with one ordinary transmitting antenna and two actuator-antennas which purpose is to reduce the electromagnetic field. It is worth stressing at this point that the purpose of this MIMO system is not to improve the capacity of transmission between the mobile unit and base station, but to predict the channel response or sense the radiated field, using the sensor antenna elements, which can then be controlled using the active control algorithms. A similar approach (not using MIMO) has been proposed for suppressing industrial frequency electromagnetic fields (power-lines, transformers and other electric equipment) [11]. The modeling of the antenna elements and the numerical electromagnetic field calculations are done in a software package called FEMLAB [12]. This modeling software is also used in combination with MATLAB to implement the adaptive algorithms used to control the electromagnetic field. The attenuation level achieved from this model suggests the possibility of using an active antenna system for this purpose. This FEMLAB model takes into consideration the actual physical material of the antenna elements and of the human head. Therefore the model also incorporates the electromagnetic coupling between the antenna elements and the human head, and between the different antenna elements.

The organisation of this paper is as follows. In Section 2, we present the FEMLAB MIMO antenna model. In Section 3, the different unconstrained adaptive algorithms used to suppress the power density of the electromagnetic field are presented. The constrained solution of the output power is derived in Section 4. Simulation results comparing these different algorithms are shown in Section 5. Finally, Section 6 concludes the paper and presents further research ideas. 


\section{THE FEMLAB MIMO MODEL}

FEMLAB is an interactive environment for modeling and simulating scientific and engineering problems by solving partial differential equations (PDE) using the finite element method [12]. One of FEMLAB applications is to solve the electromagnetic field equations. To reduce the electromagnetic field in a certain volume in space, a MIMO radio channel between the actuator antenna elements and the sensor antenna elements is modelled in order to compensate for the spatial displacement between the mobile phone and the human head. In this case FEMLAB is used to simulate the actual physical MIMO antenna system, which consists of $M$ transmitting actuator antennas and $\mathrm{N}$ receiving sensor antennas. The FEMLAB model also incorporates a physical model of the human head with the dielectric properties of the brain and skull tissues, according to the 4-Cole-Cole model [15]. In Fig. 1 we show the MIMO model for $M=3$ transmitting antennas and $\mathrm{N}=5$ receiving antennas. The operating carrier frequency of the model is set to $900 \mathrm{MHz}$ (a wave length $\lambda$ of approximately $0.33 \mathrm{~m}$ ). The spacing between the antenna elements used in this application is $0.02 \mathrm{~m}<<\lambda$; thus this arrangement can not be seen as an ordinary beamformer as the antenna elements are working in the radiated near-field.

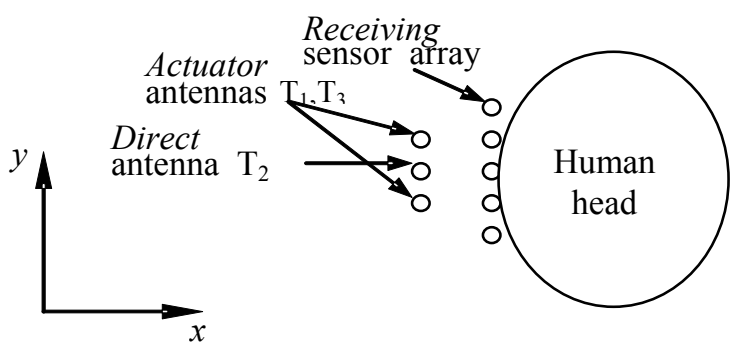

Fig. 1. A simple model of the MIMO antenna system employing $\mathrm{M}=3$ transmitting antennas and $\mathrm{N}=5$ receiving antennas.

The input signals to this MIMO antenna system are $\mathrm{M}$ separate currents in a complex-valued phasor notation, one for each transmitting antenna. The output current from the receiving antennas form an $\mathrm{N} \times 1$ complex-valued data vector denoted as the "error signal vector" $\mathbf{e}=\left[\begin{array}{lllll}e_{1} & e_{2} & e_{3} & \cdots & e_{N}\end{array}\right]^{T}$ of the system. The centre antenna $\left(\mathrm{T}_{\frac{\mathrm{M}+1}{2}}\right)$ is transmitting the signal that we want to cancel (it act as the antenna on any ordinary mobile telephone) and the M-1 flank transmitter antennas $\mathrm{T}_{\mathrm{m}}, m=1,2, \cdots \frac{M-1}{2}, \frac{M+3}{2}, \cdots M$ are denoted as actuatorantennas, which will be used to cancel the signal from the antenna $\left(\mathrm{T}_{\frac{\mathrm{M}+1}{2}}\right)$ at some specified volume in space.

Since Maxwell's equations are first order differential equations in time and space they are also linear provided that the medium for propagation is linear. In that case, we can use the space-time MIMO processing setup and the principle of superposition to form new solutions. Applying these assumptions, we can then divide the simulated radio channel from the FEMLAB MIMO model into fifteen separate SISO channels (see Fig. 2) which describe the frequency response function between each of the $\mathrm{M}$ transmitting antennas and the $\mathrm{N}$ receiving antennas. Therefore a system of $\mathrm{N}$ receiving antennas and $\mathrm{M}$ transmitting antennas can be described by the complex-valued MIMO channel frequency response matrix $\mathbf{H}$, where element $H_{N M}(\omega)$ describe the channel between transmitting antenna element $\mathrm{M}$ and receiving antenna element $\mathrm{N}$, as:

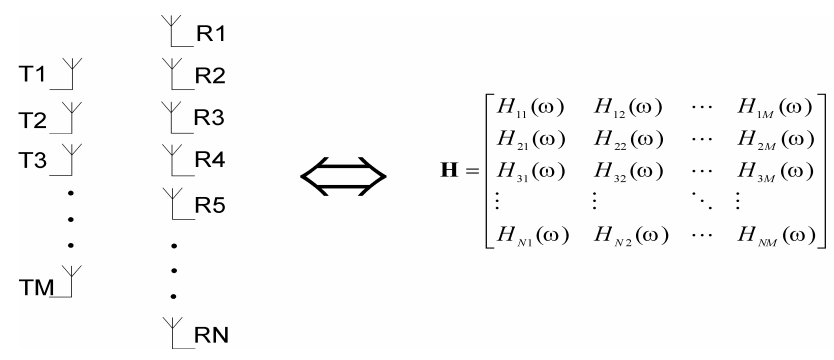

Fig. 2. The mathematical representation of the MIMO antenna system.

Partitioning the matrix $\mathbf{H}$ into separate matrices: $\mathbf{F}=\left[\begin{array}{ll}\mathbf{H}_{1} & \cdots\end{array}\right.$ $\left.\mathbf{H} \frac{M-1}{2}, \mathbf{H} \frac{M+3}{2} \cdots \mathbf{H}_{M}\right]$ for describing the actuator-antennas channel and $\mathbf{g}=\mathbf{H} \frac{M}{2}$ for describing the objective antenna channel, where $\mathbf{H}_{\mathrm{m}}$ represents a column vector $m$ of matrix $\mathbf{H}$. The system can now be described as consisting of two separate channels as depicted by blocks $\mathbf{g}$ and $\mathbf{F}$ in Fig. 3, in which the vector $\mathbf{g}$ (the direct channel) channels the "Transmitted objective signal" $s$ and the matrix $\mathbf{F}$ (the forward channel) channels the "actuator-signals" $\mathbf{x}$, which is controlled by the adaptive filter $\mathbf{w}$. The vector $\mathbf{e}$ is the residual error signal resulting from the superposition.

With the additive white Gaussian noise (AWGN) signal $\mathbf{v}$ included in the system, the residual error signal e can be expressed in matrix form as:

$\mathbf{e}=s \mathbf{g}+s \mathbf{F w}+\mathbf{v}$

where $\mathbf{w}$ is the filter vector which consists of one complexvalued filter weight for each actuator-antenna, $\mathbf{w}=\left[w_{0} w_{l}\right]^{\mathrm{T}}$.

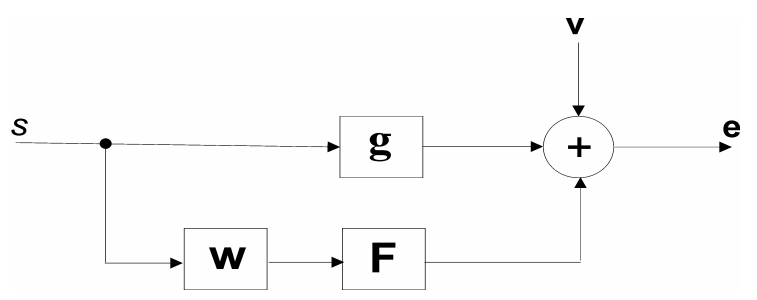

Fig. 3. Model of the direct channel $\mathbf{g}$, and the forward channel $\mathbf{F}$ controlled by the filter $\mathbf{w}$. 


\section{THE ADAPTIVE SUPPRESSION ALGORITHMS}

To get the best possible attenuation in energy sense at the receiving antenna array, the total energy output $\xi$ of the error signal e must be as low as possible. One way of achieving this task is by incorporating a complex-valued filter $\mathbf{w}$ in front of the forward channel as shown in Fig. 3. If we assume that $\mathbf{w}$ is linear and the noise is additive and Gaussian (AWGN), then the minimum residual mean energy of the error signal can be expressed as:

$$
\min _{\mathbf{w}} \xi=\min _{\mathbf{w}} \mathrm{E}\left\{|\mathbf{e}|^{2}\right\}=\min _{\mathbf{w}} \mathrm{E}\left\{\mathbf{e}^{\mathrm{H}} \mathbf{e}\right\}
$$

With the noise signal $\mathbf{v}$ included in the system, the residual error signal can be expressed as:

$$
\mathbf{e}=s \mathbf{g}+s \mathbf{F w}+\mathbf{v}
$$

If the input signal $s$ and the noise vector $\mathbf{v}$ are assumed to be uncorrelated then the mean energy of the residual error signal can be written as:

$$
\xi=\mathrm{r}_{\mathrm{g}}+\mathbf{w}^{\mathrm{H}} \mathbf{p}+\mathbf{p}^{\mathrm{H}} \mathbf{w}+\mathbf{w}^{\mathrm{H}} \mathbf{R}_{\mathrm{F}} \mathbf{w}+\mathrm{r}_{\mathrm{v}}
$$

where $\mathbf{p}$ represents the crosscorrelation between the direct channel and the forward channel, $\mathbf{R}_{\mathrm{F}}$ is the covariance of the forward channel $\left(\mathbf{R}_{\mathrm{F}}=\mathrm{s}^{*} \mathbf{F}^{\mathrm{H}} \mathbf{F} s\right), \mathrm{r}_{\mathrm{g}}$ and $\mathrm{r}_{\mathrm{v}}$ are the energy of the signal and noise, respectively. The minimum value point $\xi_{\min }$ is obtained with the complex filter weight solution $\mathbf{w}_{\text {opt }}$ :

$$
\mathbf{w}_{\mathrm{opt}}=-\mathbf{R}_{\mathrm{F}}^{-1} \mathbf{p}
$$

Equation (5) describes the Least Mean Square (LMS) solution to the problem and is the optimal solution in mean energy sense. This solution is used as a benchmark for comparing the performance of the adaptive algorithms. In this paper we present two Filtered- $X$ adaptive algorithms: the Normalized LMS (NLMS)[8-10] and the Actuator Individual $N L M S$ [10] defined, respectively, as:

$$
\begin{aligned}
& \mathbf{w}_{\mathrm{n}+1}=\mathbf{w}_{\mathrm{n}}-\left(\frac{\beta}{\operatorname{trace}\left(\mathbf{R}_{\mathbf{F}}\right)+\zeta}\right) \mathbf{x}_{n}^{H} \mathbf{e} \\
& \mathbf{w}_{\mathrm{n}+1}=\mathbf{w}_{\mathrm{n}}-\left(\frac{\left[\beta_{1} \beta_{2} \cdots \beta_{m}\right]}{\operatorname{diag}\left(\mathbf{R}_{\mathbf{F}}\right)}\right) \mathbf{x}_{\mathrm{n}}^{\mathrm{H}} \mathbf{e}
\end{aligned}
$$

where the $\beta$ 's denote step-size parameters. These algorithms were chosen because of their ability to handle non-stationary signals, and they also provided the best compromise between complexity and performance. Interested readers are referred to [8-10] for more general information about these algorithms and [13] for many other adaptive algorithms we have tested for this application.

\section{POWER CONSTRAINTS}

In the previous section we presented the unconstrained adaptive algorithms to suppress the power density of the electromagnetic field. There is however a major drawback with these adaptive algorithms: that is, although the field is attenuated by approximately $15 \mathrm{~dB}$ (as shown in plots d-f Fig. 4 in section 4 below) inside the human head, there is no control over the total output power from the mobile phone. This means that the total output power changes when the filter adapts, which is unfortunate since the magnitude of the total output power from the mobile phone depends on the distance from the base station. For example, if we take the case of three transmitting antennas and five receiving antennas, this would result in an increase of the total output power by approximately $20 \%$ (although this still gives a suppression of $15 \mathrm{~dB}$ inside the human head). However, with some other antenna spacing the mobile phone might lose the connection when the adaptive suppression filter converges towards the optimum value.

To alleviate this problem, some form of power constraint could be used on the minimization process; that is:

$\min _{\mathbf{w}^{\mathrm{H}}}\left(\mathrm{r}_{\mathrm{d}}+\mathbf{w}^{\mathrm{H}} \mathbf{p}+\mathbf{p}^{\mathrm{H}} \mathbf{w}+\mathbf{w}^{\mathrm{H}} \mathbf{R}_{\mathrm{F}} \mathbf{w}+\mathrm{r}_{\mathrm{v}}\right)$

subject to : $|s \mathbf{w}|^{2}+|s|^{2}=C, \quad C \in \mathfrak{R}$

where the symbol $\mathfrak{R}$ denotes a real number. This optimization problem can then be solved by forming a Lagrange equation [14] defined as:

$L(\mathbf{w}, \lambda)=\mathbf{w}^{\mathbf{H}} \mathbf{R}_{\mathbf{F}} \mathbf{w}+\mathbf{w}^{\mathbf{H}} \mathbf{p}+\mathbf{p}^{\mathbf{H}} \mathbf{w}-\lambda\left(C-s^{*} \mathbf{w}^{\mathbf{H}} \mathbf{w} s-s^{*} s\right)$

By differentiating this Lagrange equation and forcing it to zero, we get a suboptimal solution of $\mathbf{w}$ which is dependent on the variable $\lambda$ :

$\left(\mathbf{R}_{\mathrm{F}}+\lambda|s|^{2} \mathbf{I}\right) \mathbf{w}_{c o}=-\mathbf{p}$

where $\mathbf{w}_{\mathrm{co}}$ denote the constrained values of the filter coefficients. If we multiply equation (10) by $\mathbf{R}_{\mathbf{F}}^{-1}$ we get:

$\left(\mathbf{I}+\lambda|s|^{2} \mathbf{R}_{\mathbf{F}}^{-\mathbf{1}}\right) \mathbf{w}_{\text {co }}=-\mathbf{R}_{\mathbf{F}}^{-\mathbf{1}} \mathbf{p}$

The right hand side of equation (11) is the unconstrained optimal solution $\mathbf{w}_{\text {opt, }}$, see equation (5). Using this information and rearranging equation (11), we get:

$\mathbf{w}_{\mathbf{c o}}=-\left(\mathbf{I}+\lambda|s|^{2} \mathbf{R}_{\mathbf{F}}^{-\mathbf{1}}\right)^{-1} \mathbf{w}_{\mathbf{o p t}}$

It can be clearly seen from equation (12) that it is now possible to adjust the unconstrained solution by using a diagonal loading of the covariance matrix. The parameter $\lambda$ can be chosen so that equation (12) satisfies the constraint. Unfortunately there are no closed form solutions for the 
optimal value of the loading variable $\lambda$. However, equation (12) can be simplified by employing a Maclaurin expansion of the first term on the right hand side, for values of $\lambda$ that are close to zero:

$\left(\mathbf{I}+\lambda|s|^{2} \mathbf{R}_{\mathbf{F}}^{-\mathbf{1}}\right)^{-1} \approx \mathbf{I}-\lambda|s|^{2} \mathbf{R}_{\mathbf{F}}^{-\mathbf{1}}$

When this approximation is substituted into the solution of the constrained minimization, we get the constrained values of the filter coefficients as:

$$
\mathbf{w}_{\mathbf{c o}}=-\mathbf{w}_{\mathbf{o p t}}-\lambda|s|^{2} \mathbf{R}_{\mathbf{F}}^{-1} \mathbf{w}_{\mathbf{o p t}}
$$

To find out which value of $\lambda$ we need, the constraint in equation (8) should be solved for the value of the constrained filter coefficients $\mathbf{w}_{\mathrm{co}}$ and the required power constraint level $C$. This will yield a quadratic equation which has the following solution:

$$
\lambda=\frac{-2 \cdot \Re\left\{\mathbf{w}_{\text {opt }}^{\mathrm{H}} \mathbf{q}\right\} \pm \sqrt{\left(2 \cdot \Re\left\{\mathbf{w}_{\text {opt }}^{\mathrm{H}} \mathbf{q}\right\}\right)^{2}-4 \cdot \mathbf{q}^{\mathrm{H}} \mathbf{q}\left(\mathbf{w}_{\text {opt }}^{\mathrm{H}} \mathbf{w}_{\text {opt }}+1-\frac{C}{|s|^{2}}\right)}}{2 \cdot|s|^{2} \mathbf{q}^{\mathrm{H}} \mathbf{q}}
$$

where $\mathbf{q}=\mathbf{R}_{\mathbf{F}}^{-1} \mathbf{w}_{\text {opt }}$

So, by setting the constraining power level $C$ and using the unconstrained optimal values of the filter coefficients $\mathbf{w}_{\text {opt }}$, we can now use equation (15) to calculate what the value of $\lambda$ should be. This value is then inserted into equation (14) in order to calculate the constrained filter coefficients $\mathbf{w}_{\mathrm{co}}$.

\section{SiMULATION RESULTS}

In this section we present the results of FEMLAB simulations employing the adaptive algorithms (NLMS and Actuator Individual NLMS) MIMO antenna system and assess their effectiveness in suppressing the power density of the electromagnetic field. In addition, we compare the performance of the power constrained Actuator Individual NLMS with the unconstrained version of the algorithm. We will also include the result of employing a single transmitting antenna for use as a reference level for comparison purposes.

Fig. 4 shows that the amount of attenuation achieved by the least mean square solution is approximately $36 \mathrm{~dB}$ relative to the power level produced by a single antenna system (i.e., by using the direct transmitting antenna only as shown in Fig. 1). In addition, it is clear from this figure that the adaptive algorithms after convergence give about $23 \mathrm{~dB}$ more attenuation compared to using the five receiving antenna elements as a passive reflector. It can also be seen that the Actuator Individual NLMS is converging about $40 \%$ faster than the NLMS towards the least mean square solution since each diagonal element of the covariance matrix is normalized separately as shown in equation (7). Another interesting observation in Fig. 4 is that we can clearly see the adaptive algorithms are reaching attenuation levels that are below what can be achieved by the least square solution. The reason for this is of course that the sensor antenna elements are placed outside the human head and therefore the minimum is also located outside the human head.

In Figs. (5a) and (5b) we show the power density field for one transmitting antenna with five passive reflector elements, and three transmitting antennas tuned to the least mean square solution (i.e., the adaptive algorithms after convergence), respectively. It is evident from this surface plot that the electromagnetic power density field inside the head is lower by using the MIMO adaptive algorithms as compared to the single antenna case. In Figs. (5b) and (5c) we compare the performance of the unconstrained and power constrained MIMO adaptive algorithm respectively.

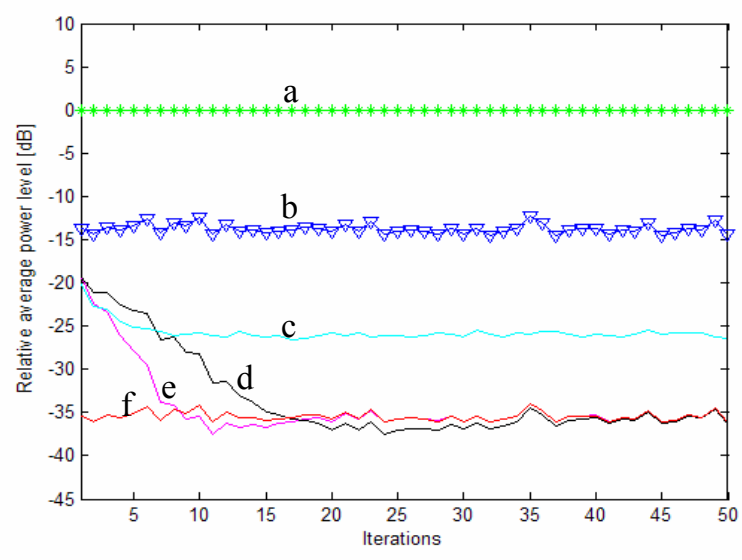

Fig. 4. The average power level inside the human head relative to the power level of a single transmitting antenna. Plots (top to bottom):
a) One transmitting antenna
b) 5 passive antenna elements (passive shielding)
c) Actuator Individual NLMS with a constraint
d) NLMS
e) Actuator Individual NLMS
f) Least mean square solution

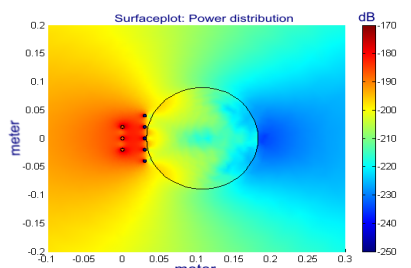

(a)

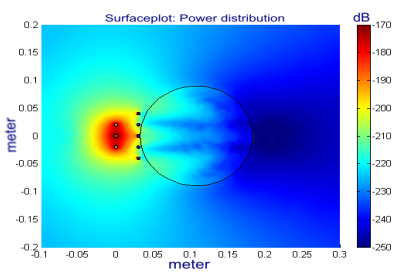

(b)

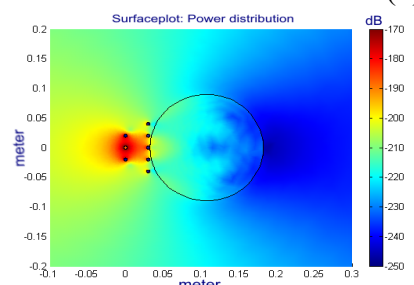

(c)

Fig. 5. Power density surface plots inside the human head (referring to Fig. 4) using: (a) one active antenna only (the center antenna), (b) three transmitting antennas tuned to the least mean square solution (unconstrained) and (c) power constrained version of (b). 
It is clear from this figure that by imposing a constraint on the minimization process we can get a compromise between the tolerated amount of total output power fluctuation and the attenuation of the power density inside the human head. This observations is also confirmed in Fig. 4 where we can see from graph $\mathrm{c}$ that in this particular case we only attain about 6 $\mathrm{dB}$ attenuation at a tolerated loss of power in the antenna farfield by approximately $20 \%$.

The behaviour of the magnitude of the converging filter coefficients $\mathbf{w}$ can be seen in Fig. 6 for the unconstrained case (red trace) as well as when the constraint is applied on the FXLMS algorithm (green trace). It can be seen from this figure that the filter coefficients will converge at the non-optimal solution that satisfy the constraint and has the shortest distance to the unconstrained optimal solution.

These initial results show the possibility of using MIMO antenna system for lowering the electromagnetic field power density.

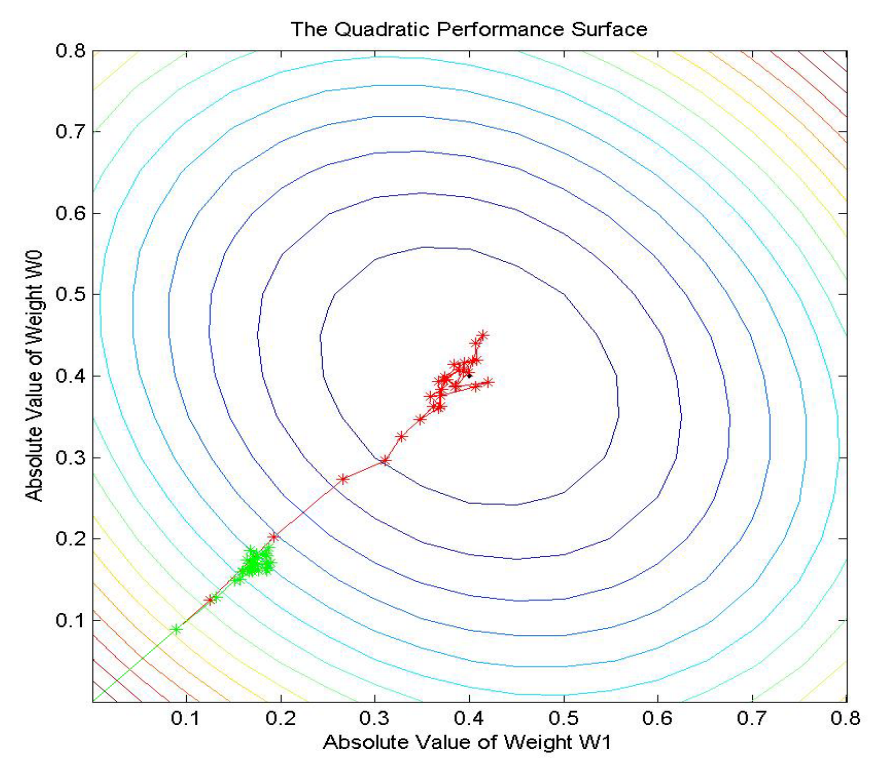

Fig. 6: An example of using the power constraint in combination with the FX-LMS algorithm. The red trace shows the convergence of the unconstrained filter coefficient magnitudes. In the green trace we have a constraint that allows for half the power needed to reach the optimal point.

\section{CONCLUSION}

In this paper we have presented a FEMLAB model which solves the partial differential equation of an electromagnetic field and simulated the physical MIMO antenna system which is controlled by various adaptive signal processing algorithms in order to suppress the field at a certain volume in space. Our simulation results have shown that the amount of attenuation achieved by using the MIMO antenna system is approximately $36 \mathrm{~dB}$ lower relative to the power level produced by a single antenna system. The attenuation level achieved from this model suggests the possibility of using an active antenna system for this purpose. We have also presented the solution for constraining the total output power of the system to a predefined level. Future work comprise of investigating the effects of the size and number of MIMO antenna elements, the overall radiation efficiency and testing the algorithms at different carrier frequencies (e.g., other GSM bands and UMTS).

\section{REFERENCES}

[1] JAMES C. LIN, "Risks to Children from Cellular Telephone Radiation" IEEE microwave magazine, march 2003.

[2] A. AHLBOM, A. GREEN, L. KHEIFETS, D. SAVITZ, A. SWERDLOW, "Epidemology of Health Effects of Radiofrequency Exposure" ICNIRP Environmental Health Perspectives, vol. 112 No. 17, dec 2004.

[3] SSI Report 2005:01, Reports from SSI:s International Independent Expert Group on Electromagnetic Fields 2003 and 2004. http://www.ssi.se/ssi_rapporter/pdf/ssi_rapp 2005 01.pdf

[4] NRPB, "Health Effects from Radiofrequency Electromagnetic Fields" Report of an Independent Advisory Group on Nonionizing Radiation: UK National Radiation Protection Board. vol. 14 No.2 2003.http://www.hpa.org.uk/radiation/publications/ documents of nrpb/pdfs/doc 14 2.pdf

[5] M.A. JENSEN, Y. RHAMAT-SAMMI, "EM interaction of handset antennas and a human in personal communications". IEEE Proceedings vol. 83, pp. 7-17, jan 1995.

[6] M. OKONIEWSKI, M.A. STUCHLY. "A study of the handset antennas and a human in personal communications". IEEE Trans. Microwave Theory, vol. 44 No. 10 oct. 1996.

[7] COST281, "Potential Health Implications from Mobile Communication Systems" Report of Scientific Comment on Individual Statements of Concern About Health Hazards of Weak EMF2001.http://www.cost281.org/activities/ hyland_comment_final23-11-2001.pdf

[8] WIDROW, B., STEAMS, S.D.: "Adaptive Signal Processing”, Prentice-Hall, 1985.

[9] KUO, S.M. and MORGAN, D.R.: "Active Noise Control Systems", John Wiley \& Sons Inc., 1996.

[10]JOHANSON, S.: "Control of Propeller-Induced Noise in Aircraft", Doctoral Thesis, Blekinge Institute of Tech., 2000.

[11]BELTZER, A., SCHENKMAN, A., SONKIN, N.: "A system of active protection from hazards of industrial frequency electromagnetic field", Electric Power Engineering, International Conference on PowerTech, Budapest, 1999.

[12]FEMLAB Reference Manual and Electromagnetics Module. COMSOL AB, 2003.

[13]HULT, T.; MOHAMMED, A.; NORDEBO, S.: "Active suppression of electromagnetic fields using a MIMO antenna system", 17th International Conference on Applied Electromagnetics and Communications, ICEC03, 2003.

[14]TIAN, Z., BELL, K.L., VAN TREES, H.L.: “A Recursive Least Squares Implementation for Adaptive Beamforming Under Quadratic Constraint", Proceedings of the $9^{\text {th }}$ IEEE Signal Processing Workshop on Statistical Signal and Array Processing", Portland, OR, pp. 9-12, September 1998.

[15]GABRIEL, C.: "Compilations of the Dielectric Properties of Body Tissues at RF and Microwave Frequencies", Brooks Air Force Technical Report AL/OE-TR-1996-0037. 


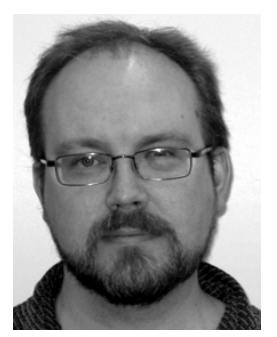

Tommy Hult was born in Sweden. Between 1986 and 1998 he was working for the Swedish Ministry of Defence as a technical analyst. He received his MSc degree in electrical engineering from Blekinge Institute of Technology in 2002. He is currently working as $\mathrm{PhD}$ student with the radio communications group at Blekinge Institute of Technology. Among his research interests are MIMO-MTMR antenna systems, wave propagation and channel modelling.

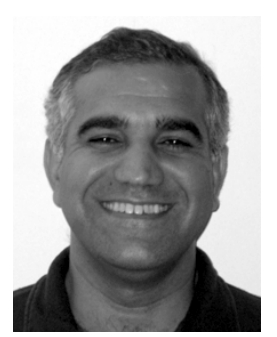

Abbas Mohammed is an Associate Professor at the Department of Signal Processing, Blekinge Institute of Technology, Sweden. He was awarded the "PhD degree" from Liverpool University, UK, in 1992 and the Swedish "Docent degree" (equivalent to 2 $\mathrm{PhD}$ degrees in different subjects) in Radio Communications and Navigation from Blekinge Institute of Technology, Sweden, in 2001. From 1993-1996, he was with the Radio Navigation Group,
University of Wales (Bangor), UK. From 1996-1998, he was with the University of Newcastle, UK, working on an EU collaborative project within the ACTS (Advance Communications Technologies and Services) Research Programme. He was also employed by Ericsson Mobile Platform, Sweden, where he consulted on power control standardisation issues for $3 \mathrm{G}$ system.

He is a Fellow of IEE, Associate Fellow of the UK's Royal Institute of Navigation, a life-member of the International Loran Association, and a member of IEEE. He is a Board Member of the IEEE Signal Processing Swedish Chapter and the Radio Engineering Journal. He is also the Swedish representative for the EU's COST 280 Action and the new COST 297 Action.

He has published many papers on telecommunications and navigation systems. He has also developed techniques for measuring skywave delays in Loran-C receivers and received the 1994 Best Paper Award from the International Loran Association, USA, in connection to this work. He was the Editor of a special issue "Advances in Signal Processing for Mobile Communication Systems" of Wiley's International Journal of Adaptive Control and Signal Processing.

His research interests are in space-time signal processing and MIMO systems, antennas and propagation, and radio navigation systems. 